\title{
Structure and kinematics of the Milky Way spirals traced by open clusters $\dagger$
}

\author{
Z. Zhu \\ Department of Astronomy, Nanjing University, China \\ email: zhuzi@nju.edu.cn
}

\begin{abstract}
Selecting 301 open clusters with complete spatial velocity measurements and ages, we are able to estimate the disk structure and kinematics of the Milky Way. Our analysis incorporates the disk scale height, the circular velocity of the Galactic rotation, the Galactocentric distance of the Sun and the ellipticity of the weak elliptical potential of the disk. We have derived the distance of the Sun to the Galactic center $R_{0}=8.03 \pm 0.70 \mathrm{kpc}$, that is in excellent agreement with the literature. From kinematic analysis, we found an age-dependent rotation of the Milky Way. The mean rotation velocity of the Milky Way is obtained as $235 \pm 10 \mathrm{~km} \mathrm{~s}^{-1}$. Using a dynamic model for an assumed elliptical disk, a clear weak elliptical potential of the disk with ellipticity of $\epsilon\left(R_{0}\right)=0.060 \pm 0.012$ is detected, the Sun is found to be near the minor axis with a displacement of $30^{\circ} \pm 3^{\circ}$. The motion of clusters is suggested to be on elliptical orbits other than the circular rotation.
\end{abstract}

Keywords. Galaxy: disk, astrometry, Galaxy: kinematics and dynamics, Galaxy: structure, open clusters and associations: general

\section{Introduction}

The young open clusters have an unusual superiority over other tracers of the disk structure in the Milky Way, providing crucial information and constraints for understanding of the Galactic kinematics and dynamics. Due to the increase of available data on open clusters in the recent years, we are able to refine our study of the Galactic structure. In this paper, we concentrate on a kinematical analysis based on proper motions, distances, radial velocities, and ages of open clusters. Considering the systematic consistency of kinematical data, we decide to use the internally homogeneous Catalogue of Open Cluster Data compiled by Kharchenko et al. (2005). In order to increase the number of clusters, we used the New Catalog of Optically Visible Open Clusters and Candidates, that includes 1689 clusters collected from the literature (Dias et al. 2002). Finally, a total amount of 301 clusters with complete spatial velocity measurements is selected for this study.

The velocity data are used to inspect the peculiar motions for the individual clusters. The components of peculiar motion are derived from the first order expansion of an asymmetric rotation of the systematic velocity field. Rejecting 22 clusters with peculiar velocities over $50 \mathrm{~km} \mathrm{~s}^{-1}$ (roughly $2.6 \sigma$ of the total velocity dispersion), 269 clusters are finally used for the kinematic analysis. Figure 1 shows the kinematical structures and distributions of 269 clusters on the Galactic plane.

$\dagger$ Supported by the National Natural Science Foundation of China(Grant Nos. 10333050 and 10673005) 

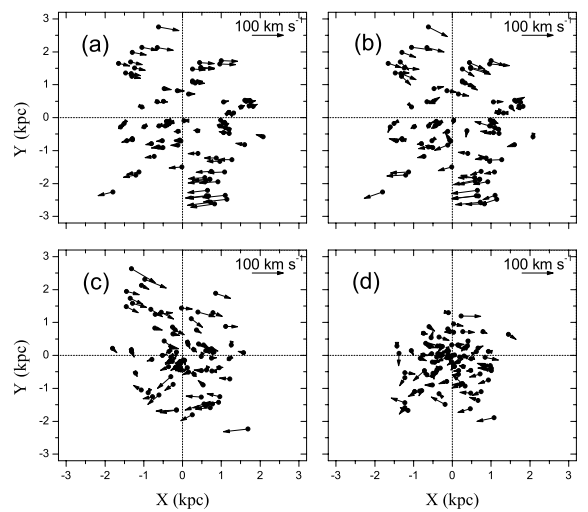

Figure 1. Observed space velocities of clusters projected on the Galactic plane. The solar motion components $u_{0}$ and $v_{0}$ are removed from the observational data. Panel (a): Simulation of 90 clusters younger than $18 \mathrm{Myr}$, assuming $R_{0}=8 \mathrm{kpc}, V_{0}=220 \mathrm{~km} \mathrm{~s}^{-1}$, and $(\partial V / \partial R)_{R=R_{0}}=4$ $\mathrm{km} \mathrm{s}^{-1} \mathrm{kpc}^{-1}$. Panel (b): 90 clusters younger than 18 Myr. Panel (c): 88 clusters with ages in the range of 18-120 Myr. Panel (d): 91 clusters older than 120 Myr.

Table 1. Solution of the parameters of the vertical distribution.

\begin{tabular}{lcccc}
\hline & \multicolumn{2}{c}{$10 \mathrm{pc}$ bins } & \multicolumn{2}{c}{$20 \mathrm{pc}$ bins } \\
\cline { 2 - 5 } & $z_{0}\lfloor\mathrm{pc}\rfloor$ & $z_{\mathrm{h}}[\mathrm{pc}]$ & $z_{0}[\mathrm{pc}]$ & $z_{\mathrm{h}}[\mathrm{pc}\rfloor$ \\
\hline all ages & $-16.1 \pm 4.0$ & $58.3 \pm 4.0$ & $-15.6 \pm 3.5$ & $56.8 \pm 3.5$ \\
$\leqslant 50 \mathrm{Myr}$ & $-12.3 \pm 4.2$ & $51.8 \pm 4.6$ & $-12.4 \pm 4.2$ & $49.6 \pm 4.4$ \\
$>50 \mathrm{Myr}$ & $-20.5 \pm 5.6$ & $67.8 \pm 5.8$ & $-19.5 \pm 5.7$ & $67.1 \pm 5.7$ \\
\hline all ages (PKRSS 2006) & $-22 \pm 4$ & $56 \pm 3$ \\
all ages (BKBS 2006) & $-14.8 \pm 2.4$ & $57.2 \pm 2.8$ \\
$\leqslant$ 200 Myr (BKBS 2006) & & $47.9 \pm 2.8$ \\
$200 \sim$ 1000 Myr (BKBS 2006) & & $149.8 \pm 26.3$ \\
\hline
\end{tabular}

\section{The disk scale height and kinematics}

The recent work concerning the structure and distribution of the Galactic open clusters is detailed by Piskunov et al.(2006, PKRSS), and by Bonatto et al.(2006, BKBS). In this Section, we first extend the study on the vertical structure based on open cluster data. In order to study the properties of clusters in different age range, we divide the sample into two subsets: young clusters with ages less than $50 \mathrm{Myr}$, and old clusters with ages larger than 50 Myr. The results are given in Table 1, where $z_{0}$ is the position about the Galactic symmetry plane, $z_{\mathrm{h}}$ is the scale height of the distribution. Note that 17 clusters associated with the Gould's Belt are removed from the samples. Comparing our results with those given by PKRSS and BKBS, we found that most of the solutions are matching very well. However, the value $z_{\mathrm{h}} \simeq 150 \mathrm{pc}$ for clusters with $200 \sim 1000 \mathrm{Myr}$ obtained by BKBS significantly deviates from our determination for older clusters. We found that the oldest clusters in BKBS have mostly erroneous distance estimate, explaining the extremal value of $z_{\mathrm{h}} \simeq 150 \mathrm{pc}$ for clusters older than $200 \mathrm{Myr}$ as derived by BKBS.

Because the objects in our sample are confined to the Galactic plane with a small scale height of $\sim 60 \mathrm{pc}$, a two dimensional asymmetric model is sufficient to describe their motion. In this case, we define the Oort's constants $A, C$ (denote the azimuthal and 
Table 2. Kinematic parameters derived from proper motions and radial velocities. The unit is in $\mathrm{km} \mathrm{s}^{-1}$ for components of the solar motion. The Oort's constants are measured in $\mathrm{km} \mathrm{s}^{-1} \mathrm{kpc}^{-1}$.

\begin{tabular}{lccccccc}
\hline & $u_{0}$ & $v_{0}$ & $w_{0}$ & $A$ & $B$ & $C$ & $K$ \\
\hline all ages (269) & $11.8 \pm 0.6$ & $13.3 \pm 0.6$ & $8.1 \pm 0.5$ & $16.4 \pm 0.9$ & $-12.9 \pm 0.9$ & $0.5 \pm 1.0$ & $-2.6 \pm 0.9$ \\
$\leqslant 50 \mathrm{Myr}(137)$ & $10.8 \pm 0.7$ & $14.6 \pm 0.7$ & $7.9 \pm 0.8$ & $16.5 \pm 0.9$ & $-14.6 \pm 0.9$ & $2.9 \pm 0.8$ & $-1.1 \pm 0.9$ \\
$>50 \mathrm{Myr}(132)$ & $12.3 \pm 0.9$ & $12.8 \pm 0.8$ & $8.2 \pm 0.8$ & $16.4 \pm 1.7$ & $-10.9 \pm 1.6$ & $-2.7 \pm 1.7$ & $-4.6 \pm 1.8$ \\
\hline
\end{tabular}

radial directions of the velocity field), $B$ (characterizes the vorticity), and $K$ (implies an overall contraction or expansion measured at the Sun). Based on proper motions, radial velocities and heliocentric distances of open clusters, these parameters including solar motions are given in (Table 2).

In an axisymmetric and stationary disk, the Oort's constants $A$ and $B$ describe a differential circular rotation of the Milky Way at the place of the Sun. Feast \& Whitelock (1997) found a low angular velocity $\left(A=14.8 \pm 0.8, B=-12.4 \pm 0.6 \mathrm{~km} \mathrm{~s}^{-1} \mathrm{kpc}^{-1}\right)$ from the Hipparcos proper motions of the Galactic Cepheids. But the majority of measurements in the recent year have shown a more or less enhanced angular velocity, including our present determination. From the Hipparcos proper motions of the Galactic O-B5 stars, Miyamoto \& Zhu (1998) derived $A=16.1 \pm 1.1$ and $B=-15.6 \pm 0.8$ in $\mathrm{km} \mathrm{s}^{-1} \mathrm{kpc}^{-1}$. From proper motions of the old red giants from ACT/Tycho-2 catalogues, Olling \& Dehnen (2003) found $A-B \simeq 32.8 \mathrm{~km} \mathrm{~s}^{-1} \mathrm{kpc}^{-1}$. According to observations of the massive compact radio source Sgr $A^{*}$ at the Galactic center by VLBA with respect to the extragalactic sources, Reid \& Brunthaler (2004) reported the apparent proper motion of $\operatorname{Sgr} \mathrm{A}^{*} \mu_{\ell}=-6.379 \pm 0.026$ and $\mu_{\mathrm{b}}=-0.202 \pm 0.019 \mathrm{mas} \mathrm{yr}^{-1}$. This apparent motion should fully reflect the Galactic rotation at the Sun, assuming Sgr A* is in rest. Then we have $A-B=-\kappa \mu_{\ell}-v_{0} / R_{0}$, where $v_{0}=5.25 \pm 0.62 \mathrm{~km} \mathrm{~s}^{-1}$ is the component of the solar motion in the direction of Galactic rotation given by Dehnen \& Binney (1998). Adopting $R_{0}=8.0 \mathrm{kpc}, A-B=29.58 \pm 0.14 \mathrm{~km} \mathrm{~s}^{-1} \mathrm{kpc}^{-1}$ was calculated. This is in excellent agreement with our determination. The present determination gives a rotation velocity $V_{0}=235 \pm 10 \mathrm{~km} \mathrm{~s}^{-1}$ from the complete sample, while $V_{0}=248 \pm 9 \mathrm{~km} \mathrm{~s}^{-1}$ is for the young and $V_{0}=218 \pm 19 \mathrm{~km} \mathrm{~s}^{-1}$ for the older sub-sample.

The precision of $R_{0}$ is directly related to many astronomical quantities, measurements and theory. According to the statistical analysis from the individual determinations by Reid (1993), $R_{0}=8.0 \pm 0.5 \mathrm{kpc}$ is currently considered as the best value, whereas the 1985 IAU standard value is $8.5 \mathrm{kpc}$. Considerable bias and uncertainties may still exist in the determinations of $R_{0}$, even if researchers have employed various efforts to improve it, e.g. from the latitude proper motion of the $\operatorname{Sgr} \mathrm{A}^{*}\left(\mu_{\mathrm{b}}=-0.202 \pm 0.019 \mathrm{mas} \mathrm{yr}^{-1}\right)$, we get $R_{0}=w_{0} / \mu_{\mathrm{b}} / \kappa=7.49 \pm 0.81 \mathrm{kpc}$, assuming Sgr A* is at rest. Here $w_{0}=7.17 \pm 0.38 \mathrm{~km} \mathrm{~s}^{-1}$ is the component of the solar motion.

Encouraged by the kinematic analysis, in which we found that the Oort's constant $A$ is independent on the cluster age, we decided to derive the Galactocentric distance of the Sun $R_{0}$. Because only small values for $C$ and $K$ of the Oort's constants are found, we are able to simply use an axisymmetric rotation model. The Oort's constant $A$ is independently derived from the proper motions of clusters. We apply this constant to constrain a kinematical model from the radial velocities

$$
v_{r}=2 A R_{0}\left(\frac{R_{0}}{R}-1\right) \sin \ell \cos b-u_{0} \cos \ell \cos b-v_{0} \sin \ell \cos b-w_{0} \sin b-\delta v_{r},
$$

where, $\delta v_{r}$ is a possible offset of the radial velocity zero-point. The parameter $2 A R_{0}$ can 
be calculated from the radial velocities. Using the constant $A$ from the proper-motion solution, the Galactocentric distance $R_{0}$ is derived in an iterative way.

The present determination of $R_{0}$, based on independent observations for proper motions and radial velocities of clusters, gives $R_{0}=8.03 \pm 0.70 \mathrm{kpc}$ that is consistent with the current "best estimate" of $R_{0}=8.0 \pm 0.5 \mathrm{kpc}$ proposed by Reid (1993).

\section{The weak elliptical distortion of the disk potential}

The persistence of a significant $K$-term or $\delta v_{r}$ for the radial velocities of Galactic young objects has been recognized before. It can be explained either as an overall kinematic contraction or expansion on the Galactic plane, or as a systematic error of the measured radial velocities. On the other hand, if the axisymmetric model is not sufficient to describe the rotation defined by the young disk stars on non-circular orbits in the Galactic plane, a non-axisymmetric model should be introduced to describe such kinematic behavior. Considering rotation velocities of clusters as a function of azimuthal angle, we find that the circular speed gradually decreases in the direction of the Galactic rotation. This fact might be an evidence for the open clusters moving on non-circular orbits. Based on the model of an elliptical disk given by Kuijken \& Tremaine (1994), the potential is expressed by

$$
\Phi(R, \phi)=\Phi_{0}(R)+\Phi_{1}(R) \cos 2\left(\phi-\phi_{\mathrm{b}}\right),
$$

where $\Phi_{0}(R)$ is the circular velocity depended axisymmetric part of the potential. In this potential, the mean tangential velocity is expressed by

$$
V_{\phi}(R, \phi)=V_{c}(R)(1-c(R) \cos 2 \phi-s(R) \sin 2 \phi),
$$

with two components of the ellipticity $c(R)=\epsilon(R) \cos 2 \phi_{\mathrm{b}}, s(R)=\epsilon(R) \sin 2 \phi_{\mathrm{b}}$. Here, $\epsilon(R)$ is the potential ellipticity with its minor axis in the direction $\phi_{\mathrm{b}}$. In order to have a more rigorous evaluation for the ellipticity of the gravitational potential, we have test various solutions by using the observed spatial velocities of clusters. Finally, we obtain the components $c\left(R_{0}\right)$ and $s\left(R_{0}\right)$. The present solution suggest

$$
\epsilon\left(R_{0}\right)=0.060 \pm 0.012, \quad \phi_{b}=30^{\circ} \pm 3^{\circ} .
$$

The present work is the first to succeed in quantifying the two elliptical components of the Milky Way potential via a consistent data set of the disk population of open clusters and based on a simple dynamic model by Kuijken \& Tremaine. Using our solution from the open clusters, the motion of objects near the Sun is suggested to be on elliptical orbits.

\section{References}

Bonatto, C., Kerber, L. O., Bica, E., \& Santiago, B. X. 1997, A $\& A 446,121$ (BKBS)

Dehnen, W. \& Binney, J. J. 1998, MNRAS 298, 387

Dias, W. S., Alessi, B. S., Moitinho, A., \& Lépine, J. R. D. 2002, A\&A 389, 871

Feast, M. W. \& Whitelock, P. 1997, MNRAS 291, 683

Kharchenko, N. V., Piskunov, A. E., Röser, S., Schilbach, E., \& Scholz, R. D. 2005, A $\& A 438$, 1163

Kuijken, K. \& Tremaine, S. 1994, ApJ 421, 178

Miyamoto, M. \& Zhu, Z. 1998, AJ 115, 1483

Olling, R. P. \& Dehnen, W. 2003, ApJ 955, 275

Piskunov, A. E., Kharchenko, N. V., Röser, S., et al. 2006, A\&̛A 445, 545 (PKRSS)

Reid, M. J. 1993, ARA\&A 31, 345

Reid, M. J. \& Brunthaler, A. 2004, ApJ 616, 872 